\title{
ARTICLE
}

\section{Energy measurement of fast neutron fields with a Recoil Proton Telescope using active pixel sensors}

\author{
Julien Taforeau $^{\mathrm{a}^{*}}$, Ingrid Schaefer ${ }^{\mathrm{a}}$, Stephane Higueret ${ }^{\mathrm{b}}$, Daniel Husson ${ }^{\mathrm{b}}$ and Lena Lebreton ${ }^{\mathrm{a}}$ \\ ${ }^{a}$ Laboratoire de Métrologie et de Dosimétrie des Neutrons, Institut de Radioprotection et de sûreté nucléaire, 13115 Saint Paul Lez \\ Durance, France; ${ }^{b}$ Radioprotection et Mesures Environnementale, Université de Strasbourg, IPHC/UMR 7178, 23 Rue du Loess \\ 67037 Strasbourg Cedex, France
}

\begin{abstract}
The spectrometer ATHENA (Accurate Telescope for High Energy Neutron metrology Applications), in development at the LNE-IRSN, characterizes energy and fluence of fast neutron fields. The detector is a Recoil Proton Telescope and measures neutron energies in the range of 5 to $20 \mathrm{MeV}$. The system is intended to become a primary standard for both energy and fluence measurements. The most innovative part of ATHENA is made of three CMOS pixel sensors, thinned down to 50 microns thickness, allowing an accurate tracking of the recoil. The use of CMOS sensors and a thick silicon diode increase the intrinsic efficiency of the detector by a factor of ten compared with conventional designs. In this paper, we demonstrate the ability of the detector to measure neutron energies in the range of 5 to $20 \mathrm{MeV}$. Experimental investigations, using mono-energetic neutron fields produced by the AMANDE facility, indicate a good reconstruction of neutron energies. The present design is still under development. The accuracy of measuring $5 \mathrm{MeV}$ and $14 \mathrm{MeV}$ neutron energies was $15 \%$ and $5 \%$, respectively.
\end{abstract}

Keywords: neutron metrology; Recoil Proton Telescope; CMOS sensors; primary standard

\section{Introduction}

The IRSN associated to the Laboratoire National de métrologie et d'Essai under the label LNE-IRSN owns the french reference for the neutron fluence, dose equivalents and kerma quantities in neutron metrology. Due to the dependence on neutron energy of the response of dosimeters, energy dependent measurements are required. The AMANDE facility (Accelerator for Metrology and Neutron Application in External Dosimetry) has been developed for this purpose, providing mono-energetic fields in the range $5 \mathrm{keV}-20$ $\mathrm{MeV}$.

Neutrons fields must be characterized using primary measurement standards with respect to energy and fluence. According to the IVM (Internal Vocabulary of Metrology), the measurement procedure used to obtain the measurand must be unrelated to a measurement standard of the same nature, i.e. the calibration of a neutron detector cannot be done using a neutron field, for instance the calibration can be achieved using alpha and protons.

Two main techniques are accessible for a primary measurement standard of fast neutron fields: the time-of-flight method [1] i.e the measurement of the neutron velocity thanks to the determination of the time

*Corresponding author. Email: julien.taforeau@irsn.fr
$T$ between the creation and the detection of neutrons. This method allows a very accurate determination on neutron energy (about $2 \%$ ) but requires a pulsed mode of accelerators. Energy measurement on continuous mode can be achieved with recoil nucleus techniques. The AMANDE facility uses for instance a BC501A liquid scintillator [2] and a SP2 spherical proportional counter [3], together with an unfolding procedure that makes it possible to measure neutrons energies. However, these detectors and methods can hardly be considered as primary standard for various reasons, the most important is the use of unfolding procedure which makes the estimation of uncertainties very complex.

At present, a new detector, based on the Recoil Proton Telescope (RPT) is under development at the LNE-IRSN, in collaboration with the RAMSES (Radioprotection et Mesures Environnementales) group of the IPHC (Institut Pluridisciplinaire Hubert Curien) [4]. The reconstruction of neutron energy, without unfolding procedure makes it possible for our system to become a primary measurement standard, for the energy and the fluence rate, in the range from $5 \mathrm{MeV}$ to 20 $\mathrm{MeV}$.

\section{Design of the detector}

The RPT device is based on the Recoil Proton 
Telescope principle to measure both the energy $\left(E_{n}\right)$ and the fluence $\left(\Phi_{n}\right)$ of monoenergetic fields.

The detection of incident neutron is possible thanks to elastic scattering in a converter, producing a nucleus recoil. A complete description of the kinematic reaction can be found in [5]. A non-relativistic approach leads to a direct relation between the energy of the incident neutron $E_{n}$ and the energy of the recoil $E_{r}$. In case of proton as recoil nucleus, the relation is given by $E_{n}=E_{p} / \cos ^{2} \theta$ where $\theta$ is the scattering angle of the proton recoil.

A low density polyethylene foil $\left(\mathrm{CH}_{2}\right)$ is used for the $\mathrm{H}(\mathrm{n}, \mathrm{p})$ reaction. The thickness of this converter $\left(25 \mu m \leq e_{0} \leq 1000 \mu m\right)$ can be chosen freely depending on the requested uncertainty for energy measurement. The tracking of the recoil is performed with three silicon CMOS pixel sensors (MIMOSTAR3L [6]). Each sensor is composed of $320 \times 320$ pixels with a pitch of $30 \times 30 \mu \mathrm{m}^{2}$. The use of three sensors is motivated by the possibility to use a better coincidence criterion for discrimination of tracks than with only two sensors. However, increasing the number of CMOS sensors decrease the energy range of the telescope, consequently, three sensors appears to be the best solution for our detector. The residual energy of the recoil is collected in a $3 \mathrm{~mm}$ thick $\mathrm{Si}(\mathrm{Li})$ diode designed to stop $20 \mathrm{MeV}$. with a resolution of $40 \mathrm{keV}$.

\subsection{Energy measurement procedure}

The reconstruction of $E_{p}$ is possible by measuring the energy in the diode $E_{d}$ and adding the proton energy losses $\Delta E_{\text {cmos }}$ in the three CMOS sensors and in the converter $\Delta E_{\text {conv }}$, leading to the $\hat{E}_{p}$ estimator.

$$
\hat{E}_{p}=E_{d}+\Delta E_{\text {cmos } 3}+\Delta E_{\text {cmos } 2}+\Delta E_{\text {cmos } 1}+\Delta E_{\text {conv }}
$$

The energy statistical fluctuations of the recoil is not included in Eq. (1) but it appears later in the uncertainty calculation. The estimation of energy loss of recoil in converter is calculated assuming an interaction of neutron in the middle of the converter.

The scattering angle is estimated thanks to the CMOS sensors performing the tracking of the proton. This tracking system provides the coordinates of the recoil in each CMOS sensor. Taking $\left(\mathrm{X}_{2}, \mathrm{Y}_{2}\right)$ in the second plane and $\left(\mathrm{X}_{1}, \mathrm{Y}_{1}\right)$ in the first one, we define the scattering angle estimation $\hat{\theta}$ as the angle $\theta_{12}$, corresponding to the angle between CMOS 1 and 2, with $d$ the distance between sensors 1 and 2 . Considering $\hat{\theta}$ as $\theta_{13}$ or $\theta_{23}$ is also possible but less accurate because of Multiple Coulomb Scattering (MS) in CMOS layers.

\subsection{Interest of the present design}

Conventional telescopes are usually used as primary measurement standard for fluence measurement and a functioning as spectrometer is possible but with a poor resolution. The spectrometer ATHENA presents some additional elements which makes the energy measurement more accurate and the detection efficiency higher. The CMOS sensor used in the present design were initially designed for the STAR collaboration to detect Minimum Ionizing Particle. Individual pixels are read at $20 \mathrm{MHz}$ frequency, and the readout time for a complete frame is then $5 \mathrm{~ms}$. The digital information with a pitch of 30 microns leads to a spatial resolution about of $8.6 \mu \mathrm{m}$ asssuming an uniform distribution of proton recoil. This tracking introduces a complexity in the estimation of the fluence measurement but allows a more accurate reconstruction of the neutron energy, thanks to the estimation of the scattering angle. The relatively thin CMOS sensor $(50 \mu \mathrm{m})$ limits both the Multiple Scattering $\sigma_{M S}^{M C N P X} \approx 32 \mathrm{mrad}$ at $14 \mathrm{MeV}$ according to an MCNPX calculation of proton scattering in CMOS layer) and the energy loss of the recoil in the device. Moreover, if conventional designs are limited to track protons scattered at $0^{\circ}$, the geometry of our detector allows a solid angle coverage up to $41^{\circ}$. As a consequence the intrinsic efficiency of our detector is increased by a factor of 10-100 compared to zero angle devices like the one used at the PTB [7]. In term of energy reconstruction, our device is likely to reconstruct mono-energetic neutron beams with an accuracy about of $15 \%$ at $5 \mathrm{MeV}$ and $5-8 \%$ for higher energy, these performances are similar to conventional designs.

\section{Energy measurements}

The energy range measurement of the device $[5 \mathrm{MeV}, 20 \mathrm{MeV}]$ is evaluated at three energies $(5,14$ and $17 \mathrm{MeV}$ ) with both Monte-Carlo simulations and experimental campaigns. The entire geometry of the telescope is studied with MCNPX v2.6. The incident neutron beam is considered as perfectly monoenergetic and it hits the entrance window perpendicularly.

\subsection{Monte Carlo validation}

\subsubsection{Performances of the reconstruction}

The data analysis program (DAP) is compared to an MCNPX calculation. The difference observed between the DAP and MCNPX leads an estimation of energy measurement performances. The reconstruction is unbiased for a thin converter, in this case, the procedure estimates the neutron with a very good precision. For instance a converter foil of $e_{0}=50 \mu \mathrm{m}$ involves a dispersion on $\mathrm{E}_{\mathrm{n}}$ of 2.9, 1.8 and $1.2 \%$ at 5, 14 and $17 \mathrm{MeV}$ respectively. However, a thick converter introduces a bias in the reconstruction; the hypothesis of uniformity of the proton creation inside the converter is not verified anymore. For thick converter, the probability density function for the interaction of a neutron at a depth $\mathrm{z} e^{(-\Sigma z)}$ has to be multiplied by the probability for the proton to escape the converter $p\left(z, e_{0}, E_{p}\right)$. 


\subsubsection{Background measurement}

The background measured in the silicon diode is due to neutron induced reactions with the detector environment. A complete description of the background hits is provided, including $(n, \gamma),(n, \alpha),(n, p)$ and $(n, x)$ reactions. Results of simulations are compared to experimental spectra obtained at the AMANDE facility (Figure 1) for $14 \mathrm{MeV}$ neutrons. Above $2 \mathrm{MeV}$, a very good agreement is found between calculations and experiments. The silicon diode, due to its $3 \mathrm{~mm}$ thickness, is responsible for the major contribution to pollution. For instance, $77 \%$ of the false proton signal is due to $\mathrm{Si}(\mathrm{n}, \mathrm{p})$ reaction in the diode, and to a lesser extent, the Printed Circuit Board induces a proton pollution of $11 \%$. Below $2 \mathrm{MeV}$, the diode signal is dominated by the reaction $(n, \gamma)$ producing photoelectrons $(85 \%)$ and the elastic scattering on silicon $\mathrm{Si}(\mathrm{n}, \mathrm{Si})(15 \%)$. Up to 2 $\mathrm{MeV}$, the two main reactions are the $\operatorname{Si}(n, p)$ and $\operatorname{Si}(n, \alpha)$ for a contribution to respectively $63 \%$ and $33 \%$ of the global signal. Moreover, the proton recoil signal contributes for only $0.2 \%$ (Cf. Figure 1$)$.

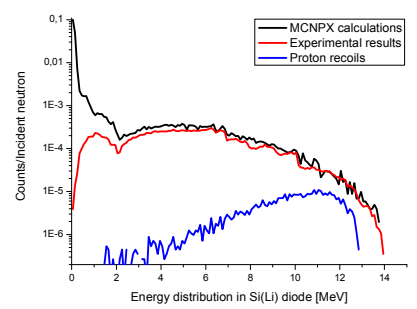

Figure 1. Comparison of energy distribution in $\mathrm{Si}(\mathrm{Li})$ diode (notice the log scale) between MCNPX calculations (black curve) and experimental data (red curve) for $14 \mathrm{MeV}$ neutron and $500 \mu \mathrm{m}$ converter, (the expected distribution of true proton recoils is indicated by blue curve).

\subsection{Experimental measurements}

\subsubsection{Experimental set-up}

Experimental campaigns are performed at the AMANDE facility. The device is studied at three energies (5, 14 and $17 \mathrm{MeV})$ via the reactions ${ }^{2} \mathrm{H}(\mathrm{d}, \mathrm{n}){ }^{3} \mathrm{He}$ and ${ }^{3} \mathrm{H}(\mathrm{d}, \mathrm{n}){ }^{4} \mathrm{He}$. The device is placed at $0^{\circ}$ for 5 and $17 \mathrm{MeV}$ and $98^{\circ}$ from the target for $14 \mathrm{MeV}$.

\subsubsection{Data analysis}

A candidate for recoil a proton event is validated if a 4-coincidences track is recorded, ie if a signal is detected in the three CMOS layers and in the diode. Under this condition, the energy distribution of such candidates is given in Figure 2.

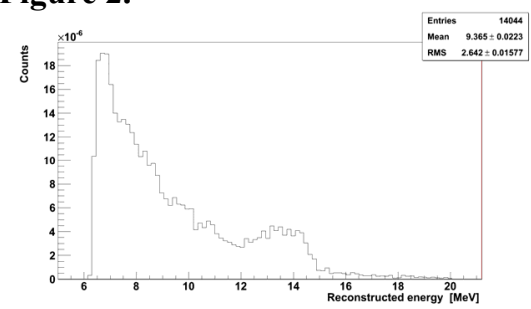

Figure 2. Energy reconstruction of recoil proton candidates for $14 \mathrm{MeV}$ neutron and a $500 \mu \mathrm{m}$ converter.
The large amount of pollution in the diode implies the identification of fake events as proton recoil events. A better discrimination candidate/proton recoil is possible acting on three characteristics of tracks: 1) The estimation of the scattering angle $\theta, 2$ ) The deposited charge in CMOS layers and 3) The goodness of the tracking by using a $\chi^{2}$ criterion. The set of criteria is sufficiently general to be considered as independent of the neutron energy to measure.

1. The theoretical model for $(n, p)$ scattering predicts a quasi-uniform distribution of the scattering angle from 0 to $180^{\circ}$ in the center-of-mass. The geometry of our device constrains the detection solid angle and consequently the angle $\theta$ between 0 and $41^{\circ}$ in the laboratory. In addition, the Multiple Scattering degrades slightly the precision on the estimation of $\theta$. The comparison between $\theta_{12}$ and $\theta_{23}$, presented in Figure 3, leads to the estimation of $\sigma_{M S}^{\exp }=\sigma_{\left(\theta_{12}-\theta_{13}\right)} \approx 27 \mathrm{mrad}$, which is the difference of two estimators of the scattering angle $\theta_{12}-\theta_{13}$. This value is in good agreement with our MCNPX calculation. The first discrimination criterion is consequently $\hat{\theta}<0.7 \mathrm{rad}$ and $\theta_{12}-\theta_{23}<0.05 \mathrm{rad}$.

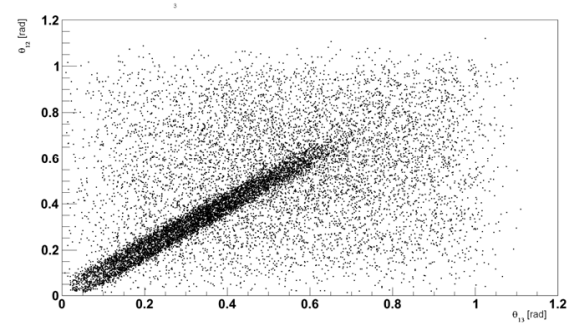

Figure 3. Correlation between two estimators $\left(\theta_{13}, \theta_{12}\right)$ of the scattering angle $\theta$.

2. The CMOS sensors do not measure exactly the energy losses of particle in the layer. However, this information can be used as a discriminator between weakly ionizing particle, ie photoelectrons, and hardly ionizing particles such as recoil protons. Unfortunately, the information on the deposited charge (ADC) provided by the present design of CMOS layers is not accurate. A calibration of this charge is in progress with a well known proton beam. Despite this limitation, discrimination between proton and other particles is possible, and is chosen as a second criterion.

3. The last criterion indicates if the candidate for proton recoil is in good agreement with a model of linear trajectory. The acceptable $\chi^{2}$ in our experimental estimations gives $0.2 \leq \chi^{2} \leq 10$. The lower limit defines the discrimination photoelectron/proton recoil and the upper limit distinguishes the fortuitous coincidence associated to a non linear propagation.

\subsubsection{Results and discussion}

The application of this set of criteria allows the discrimination of recoil proton events from fortuitous 
coincidences. Figure 4 presents the energy estimation of monoenergetic field produced by AMANDE. A comparison with Figure 2 indicates that $96 \%$ of candidates for proton events are considered as pollution and only $4 \%$ satisfy discrimination criteria.

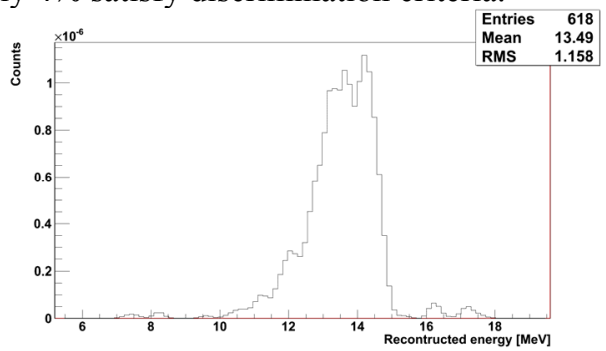

Figure 4. Estimation of the neutron energy distribution, normalized by the fluence rate $\varphi_{n}$ at the entrance window of the detector, after reconstruction and elimination of background, the expected energy is $14 \mathrm{MeV}$.

Finally, this analysis estimates the principal peak at $\mathrm{E}_{\mathrm{n}}=13.8 \mathrm{MeV}$, few events are present below $14 \mathrm{MeV}$ and can be considered as secondary peaks at $12 \mathrm{MeV}$ and $8 \mathrm{MeV}$. However, few events around $17 \mathrm{MeV}$ are reconstructed, these events are due to an overestimation of energy losses of recoil protons in the polyethylene foil. Additional measurements at 5 and $17 \mathrm{MeV}$ gives after reconstruction $4.7 \mathrm{MeV}$ and $16.9 \mathrm{MeV}$. Experimental results indicated a systematic bias of the reconstruction order of $-6 \%,-1.4 \%$ and $-0.5 \%$ at 5,14 and $17 \mathrm{MeV}$ respectively

\subsubsection{Uncertainties on energy}

All uncertainties sources are included in the calculation: due to the calibration in the diode, the device geometry, the Multiple Coulomb Scattering, the calculation of proton energy losses (particularly the influence of the converter thickness), the uncertainty of the proton tracking due to the pixel pitch of CMOS sensors. The calculation is performed by Monte Carlo propagation, results are listed in Table 1 at $5 \mathrm{MeV}, 14$ and $17 \mathrm{MeV}$ considering different converter thicknesses. A sensitivity analysis indicates that the most influent parameters are the calculation of proton energy losses in the converter foil the measurement of the residual energy in the diode.

Table 1. Uncertainties measurements of neutron energy, results are indicated in percent as a variation coefficient $(\sigma / \mu)$ according to a Gaussian fit.

\begin{tabular}{|c|c|c|c|}
\hline $\begin{array}{c}\text { Converter } \\
\text { thickness }[\mu \mathrm{m}]\end{array}$ & $5 \mathrm{MeV}$ & $14 \mathrm{MeV}$ & $17 \mathrm{MeV}$ \\
\hline 20 & $5.5 \%$ & - & - \\
\hline 50 & $6.0 \%$ & - & - \\
\hline 200 & - & $5.6 \%$ & $5.4 \%$ \\
\hline 500 & - & $7.5 \%$ & $6.4 \%$ \\
\hline 750 & - & $9.9 \%$ & $8.0 \%$ \\
\hline
\end{tabular}

\section{Conclusion}

Experimental results demonstrate the ability of our system to reconstruct the energy of monoenergetic neutron fields. The device, after background identification, measures the neutron energy in the range [5 MeV-20 MeV] with a precision about of 5-10\% for energy up to $14 \mathrm{MeV}$ and $10-15 \%$ for lower energies. The validation of the reconstruction is performed thanks to MCNPX calculation; a complete identification of the various sources of pollution is presented which motivates the 4-coincidences criteria for the recoil proton tracking.

\section{Acknowledgments}

This work was supported by the French National Laboratory of Metrology and the IRSN, in the frame of the LNE-IRSN association. We wish to thank the LMDN staff and especially, V.Gressier, A.Martin and M.Pepino, for providing the beam and their assistance in the preparation of the experiment.

\section{References}

[1] M.A. Cognet and V. Gressier, Development of a measurement reference standard for neutron energies between $1 \mathrm{MeV}$ and $20 \mathrm{MeV}$ using time of flight method at the AMANDE facility, Metrologia 47 (2010), pp. 377-386.

[2] H. Klein, Neutron spectroscopy in mixed fields: NE213/BC501A liquid scintillation spectrometer, Rad. Prot. Dos. 107 (2003), pp. 95-109.

[3] G. Pichenot, S. Guldbake, B. Asselineau, V. Gressier, C. Itié, H. Klein, K. Knauf, L. Lebreton, S. Löb, L. Pochon-Guerin, D. Shlegel and W. Sosaat, Characterisation of recoil recoil proportional counters used for neutron spectroscopy, Nucl. Inst. Meth. A 476 (2002), pp. 165-169.

[4] J. Taforeau, S. Higueret, D. Husson, L. Lebreton, T.D. Le and M. Petit, A new Recoil Proton Telescope for characterization of energy and fluence of fast neutron fields, Proc. JINST 7 (2012), FNDA, Israël, Nov. 6-11.

[5] D.J. Thomas and E.J. Axton, The need for a relativistic approach when calculating proton recoil telescope efficiencies, Nucl. Inst. Meth. A 174 (1980), pp. 321-322.

[6] Y. Gornushkin, G. Claus, W. Boer, J. Bol, G. Deputch, A. Dierlamm, W. Dulinski, D. Husson, M. Koppenhöfer, J.L. Riester and M. Winter, Test results of monolithic active pixel sensor for charged particle tracking, Nucl. Inst. Meth. A 478 (2002), pp. 311-315.

[7] B.R.L Sieber, H.J Brede, H. Lesiecki and M. Cosack, Corrections and uncertainties for neutron fluence measurements with proton recoil telescopes in anisotropic fields, Nucl. Inst. Meth. A 235 (1985), pp. 542-552. 